\title{
Cirugías con cinchas medio uretrales para el tratamiento de la incontinencia urinaria de esfuerzo femenina
}

Mid-urethral sling operations for stress urinary incontinence in woman

\section{Objetivos}

Evaluar los efectos clínicos del tratamiento quirúrgico con cinchas medio uretrales (CMU) o "slings" para las mujeres con incontinencia urinaria de esfuerzo (IOE), de esfuerzo urodinámica (IOEU) o mixta (IOM).

\section{Fuentes de datos}

Se realizó la búsqueda en el Registro Especializado del Grupo Cochrane Incontinencia, que revisa periódicamente CENTRAL, MEDLINE, EMBASE y registros de ensayos clínicos. Además se realizó una búsqueda manual de resúmenes de conferencias y se revisó la bibliografía de los principales artículos incluidos.

\section{Selección de estudios}

Se incluyeron ensayos clínicos controlados aleatorizados cuasialeatorizados sobre IOE, IOEU o IOM en los cuales ambas ra-
Ford AA y col. Cochrane Database of Systematic Reviews 2017, Issue 7. mas de tratamiento involucraran cirugías con CMU.

\section{Extracción de datos}

Los datos fueron extraídos por dos revisores independientes, quienes además evaluaron el riesgo de sesgo de los ensayos utilizando el sistema GRADE.

\section{Resultados Principales}

Se incluyeron 81 ensayos con un total de 12.113 mujeres. Se evaluaron los resultados y complicaciones de las cirugías con vías de abordaje retropúbica (RP) y trans-obturadora (TOT). Los resultados principales se detallan en tabla 1. La calidad de vida fue evaluada por 11 estudios con 16 cuestionarios validados diferentes, por lo que no se realizó meta-análisis. Todos los estudios, excepto uno, reportaron mejoría luego de intervención (independientemente de la vía).

Tabla 1. Comparación de la efectividad y los efectos adversos de las cirugías con cinchas medio uretrales en el tratamiento de la incontinencia urinaria de esfuerzo, de esfuerzo urodinámica o mixta.

\begin{tabular}{|c|c|c|c|c|c|}
\hline Desenlace & Via retropúbica & $\begin{array}{c}\text { Via } \\
\text { trans-obturadora }\end{array}$ & Efecto relativo (IC 95\%) & $N^{\circ}$ de pacientes & $\begin{array}{l}\text { Calidad de la evidencia } \\
\text { (GRADE) }\end{array}$ \\
\hline Curación subjetiva a corto plazo (<1 año) & 844 por 1000 & 827 por 1000 & $\operatorname{RR} 0,98(0,96$ a 1$)$ & 5514 & Moderada \\
\hline Curación subjetiva a largo plazo (>5 años) & 707 por 1000 & 671 por 1000 & $\operatorname{RR} 0,95(0,87$ a 1,04$)$ & 714 & Moderado \\
\hline Perforación vesical o uretral & 49 por 1000 & 6 por 1000 & $\operatorname{RR} 0,13(0,08$ a 0,20$)$ & 6372 & Moderado \\
\hline Disfunción del vaciado (0 a 5años) & 72 por 1000 & 38 por 1000 & $\operatorname{RR} 0,53(0,43$ a 0,65$)$ & 6217 & Moderado \\
\hline Urgencia de novo o $10 U^{\star}$ (<1 año) & 82 por 1000 & 80 por 1000 & $\operatorname{RR} 0,98(0,82$ a 1,17$)$ & 4923 & Moderado \\
\hline Dolor inguinal & 14 por 1000 & 66 por 1000 & $\operatorname{RR} 4,62(3,09$ a 6,92$)$ & 3226 & Moderado \\
\hline Dolor suprapúbico & 29 por 1000 & 8 por 1000 & RR 0,29 (0,11 a 0,78) & 1105 & Moderado \\
\hline Erosión de malla hacia vagina ( 0 a 5 años) & 20 por 1000 & 22 por 1000 & RR $1,13(0,78$ a 1,65$)$ & 4743 & Moderado \\
\hline Re-intervención por falla ( $<1$ año) & 19 por 1000 & 31 por 1000 & $\operatorname{RR} 1,64(0,85$ a 3,16$)$ & 1402 & Moderado \\
\hline Re-intervención por falla (>5 años) & 11 por 1000 & 100 por 1000 & RR 8,79 (3,35 a 23) & 695 & Baja \\
\hline
\end{tabular}

*IOU: incontinencia de orina de urgencia

\section{Conclusiones}

Las cirugías con CMU constituyen la intervención anti-incontinencia más estudiada en la historia. Son procedimientos altamente efectivos en el corto, mediano o largo plazo, indepen- dientemente de la vía de acceso. Mejoran significativamente la calidad de vida. En la vía trans-obturadora, no hay diferencias entre sus dos diferentes técnicas. En la vía retropúbica, la técnica "de abajo hacia arriba" es más efectiva.

\section{Comentario}

El estudio resumido en este artículo es el primer meta-análisis que evalúa efectividad a largo plazo de las cirugías con CMU. Confirma que son muy efectivas luego de 5 años de seguimiento, independientemente de la vía de acceso. A pesar de ello, debemos aguardar datos a más largo plazo por dos razones. Primero, porque la tasa de re-operación por falla a mediano-largo plazo es mayor en los TOT; y segundo, porque los resultados de estudios observacionales con más de 15 años de seguimiento (no incluidos en este meta-análisis) muestran un lento declinamiento de la efectividad de las cirugías "dependiente del tiempo". Este evento no ha podido ser confirmado ni descartado por los ensayos analizados en este meta-análisis, porque el seguimiento máximo no excedió los 10 años. Con respecto a las complicaciones, las cirugías comparadas presentan una incidencia muy baja de eventos y sin diferencias estadísticamente significativas, excepto por el dolor postoperatorio, que es mayor en los TOT, pero que raramente excede las 8 semanas y es fácilmente manejable con analgésicos. Excepto por las relacionadas con exposición de malla, las demás complicaciones también son observadas en las cirugías clásicas. Lamentablemente, no se dispone de análisis de subgrupos que permitan comparar la información relacionada con la presencia de déficit esfinteriano intrínseco, hipermovilidad uretral, obesidad, el antecedente de cirugías previas, la presencia o ausencia de prolapso, la experiencia del cirujano, etc. La ausencia de estos datos le resta riqueza para el uso clínico de los resultados obtenidos por los revisores.

\section{Conclusiones del comentador}

Las cinchas medio uretrales comparadas en este estudio son altamente efectivas para el tratamiento de la incontinencia de orina de esfuerzo. Lamentablemente este estudio no aclara si una técnica es superior a la otra (RP vs TOT) en el tratamiento de subgrupos especiales de pacientes (obesas, mayor edad, con déficit esfinteriano intrínseco, entre otros).

Adrián Saavedra Sánchez [ Servicio de Ginecología. Sección Uroginecología. Hospital Italiano de Buenos Aires. adrian.saavedra@ hiba.org.ar ]

Saavedra Sánchez A. Cirugías con cinchas medio uretrales para el tratamiento de la incontinencia urinaria de esfuerzo femenina. Evid Act Pract Ambul. 2017. 20(4):94. Comentado de: Ford A y col. Mid-urethral sling operations for stress urinary incontinence in women. Cochrane Database of Systematic Reviews 2017, Issue 7:CD006375. 